\title{
SPECIAL X-RAY SILICON DETECTORS FOR MEDICAL APPLICATIONS
}

\author{
E. Halmagean \\ National R\&D Research Institute for Microtechnology \\ Str. Erou Iancu Nicolae 32B, 72996 Bucharest, Romania \\ D. WAGNER \\ "Politechnica" University, Bucharest, Romania \\ E. Tsoi, K. Misiakos \\ Institute of Microelectronics, National Centre for Scientific Research "Demokritos" \\ 15310 Ag. Paraskevi, Attiki, POB 60228, Greece \\ M. Ohanisian, A. Veron \\ Baneasa S.A., Str. Erou Iancu Nicolae 32, 72996 Bucharest, Romania

\section{Cimpoca and D. Lazarovici} \\ Institute of Atomic Physics, 76900 Bucuresti-Magurele, Romania \\ The paper presents some results concerning the technology and be- \\ haviour of X-ray silicon detectors intended for controlling low radiation levels \\ in interventional cardiology. An improved planar process was used for ob- \\ taining large area guard-ring test structures. The electrical characteristics, \\ their modification after exposure to radiation and the results of spectroscopic \\ $\mathrm{X}$-ray measurements are discussed.
}

PACS numbers: $07.85 .-\mathrm{m}, 61.80 . \mathrm{Cd}$

\section{Introduction}

For several years, modern semiconductor detectors have been the primary choice for the measurement of nuclear radiation in various scientific fields [1]. Nowadays the recently developed high resolution silicon detectors find their way in medical applications [2]. As a consequence, many efforts have been devoted to the development of high sensitivity radiation hardened X-ray detectors for the energy range 10-100 keV [3]. The paper presents an improved technology for obtaining low noise silicon detectors which could be used in synchrotron radiation experiments. The test structures were electrically characterized before and after exposure to specific X-ray doses between $10-10^{6} \mathrm{~Gy}$. These detectors were used for the measurement of the ${ }^{241} \mathrm{Am}$ spectrum in the energy range $10-60 \mathrm{keV}$. The results indicated that the proposed process enables the development of reliable silicon detectors to be used in controlling the low radiation levels encountered in medical and synchrotron radiation applications. 


\section{Experimental}

The test structures were fabricated on $n$-type high purity silicon wafers produced by Topsil having a resistivity of about $4500 \Omega \mathrm{cm}$. The devices consisted of square geometry $p n$ diodes with diffused guard rings equipped with separate contacts. Two different active areas were analyzed: a small diode D1 $\left(3 \times 3 \mathrm{~mm}^{2}\right)$ and a large diode D2 $\left(5 \times 5 \mathrm{~mm}^{2}\right)$. The 3 inch diameter wafers were first oxidized at $1050^{\circ} \mathrm{C}$ in dry oxygen with $2-3 \%$ trichloroethylene in order to obtain a high quality initial oxide on the silicon surface. The oxidation step was followed by an in situ high temperature $\mathrm{N}_{2}$ annealing to reduce the interface charges. After the first photoengraving step, boron was implanted simultaneously in the active area and in the guard ring. The implantation energy was $40 \mathrm{keV}$ and the dose $10^{14} \mathrm{~cm}^{-2}$. A second phosphorous implantation was performed on the rear side of the silicon wafers with an energy of $80 \mathrm{keV}$ and a dose of $10^{16} \mathrm{~cm}^{-2}$. The implanted impurities were first activated at $900^{\circ} \mathrm{C}$ in $\mathrm{N}_{2}$ and subsequently driven in at $1000^{\circ} \mathrm{C}$. The diffusion phase comprised two steps: the first one in dry oxygen with 2-3\% TCE and the second one in pure $\mathrm{N}_{2}$. The resulting boron profile in the active area of the detectors was determined by the spreading resistance method. A 0.5 micron layer of $\mathrm{SiO}_{2}$ was next deposited on the wafers in an LPCVD hot wall reactor using TEOS as the source. The final thermal treatment was performed at $850^{\circ} \mathrm{C}$ in $\mathrm{N}_{2}$, followed by a very slow cooling to $600^{\circ} \mathrm{C}$ for gettering the unwanted impurities from the silicon active zones. The contact windows were opened in the central diode area and in the guard ring by standard photolithography. An aluminium layer 1 micron thick was deposited on the wafer surface by thermal evaporation.

The reverse electrical characteristics of the detector test structures were measured with a Keithley 616 ammeter and the typical ones are presented in Fig. 1. The $C-V$ characteristics were recorded at $1 \mathrm{MHz}$ on a PARC $410 \mathrm{C}-\mathrm{V}$ Plotter system and are presented in Fig. 2. The reverse currents of the devices were mea-

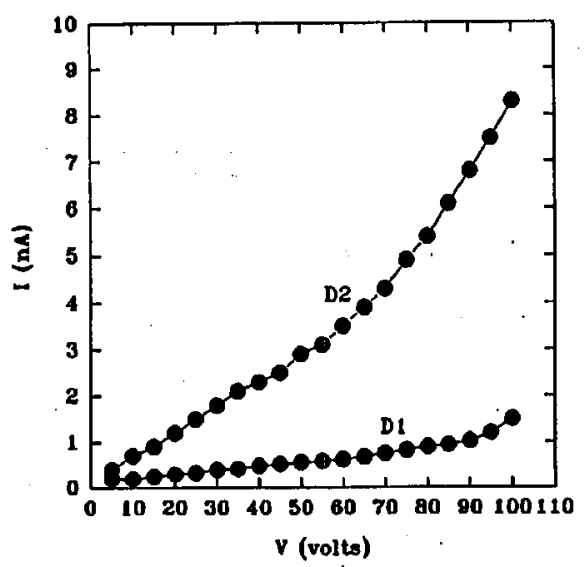

Fig. 1. $I_{R}-V_{R}$ characteristics of the small area (D1) and large area (D2) X-ray detector test structures. 


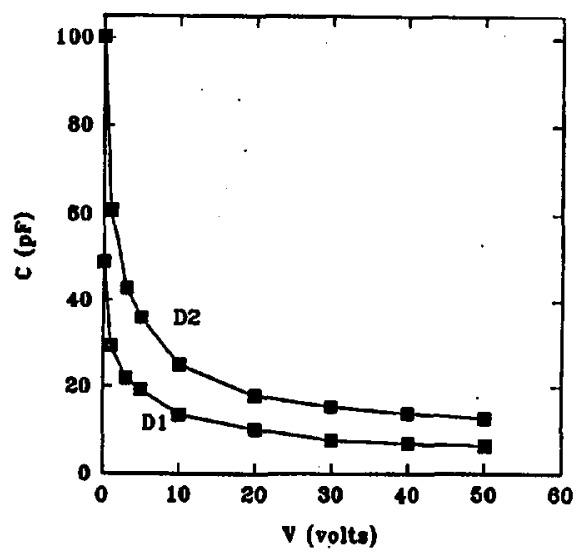

Fig. 2. $C-V_{R}$ characteristics of the detector test structures.

sured after specific radiation doses in the range $10-10^{6} \mathrm{~Gy}$ in order to evaluate the degradation of their electrical characteristics. Some large area detectors were also used for spectroscopic X-ray measurements on an ORTEC setup. The spectrum of ${ }^{241} \mathrm{Am}$ was recorded at room temperature in the energy range $10-60 \mathrm{keV}$.

\section{Results and discussion}

The measured electrical characteristics of the test structures demonstrated that the proposed processing scheme could provide very small reverse leakage currents on large area devices and is suitable for producing sensitive $\mathrm{X}$-ray detectors to be used in synchrotron radiation experiments. The stability of the reverse leak-

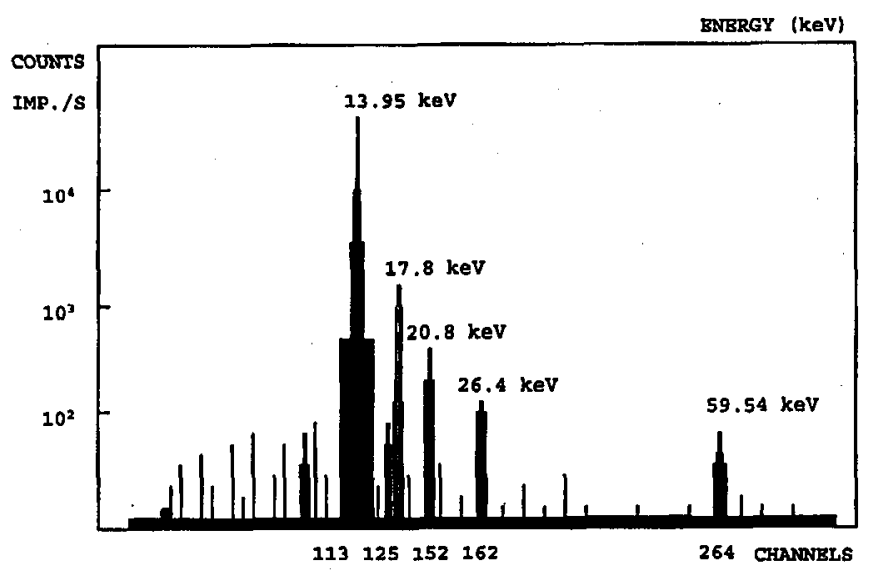

Fig. 3. The measured ${ }^{241} \mathrm{Am}$ spectrum for a test-structure with guard ring: $2.87 \mathrm{keV}$ resolution, $2.44 \mathrm{keV}$ noise. 
age currents after detector exposure to different radiation doses was studied. The results showed that after approximately $10^{5} \mathrm{~Gy}$ the leakage current of the tested devices begins to gradually increase. The main reason for this degradation is considered to be the interface states and oxide traps generation due to the ionizing radiation [4]. This dose is high enough to enable the use of the detectors for controlling low radiation levels in medical applications. The recorded spectrum of ${ }^{241} \mathrm{Am}$ is depicted in Fig. 3. The reverse voltage of $40 \mathrm{~V}$ was selected in order to drastically reduce the leakage current of the diode and to correspondingly improve the signal to noise ratio of the detector. The test structure succeeded to distinguish 5 peaks with a resolution of about $3.135 \mathrm{keV}$.

\section{Conclusions}

The proposed processing sequence enabled the development of low noise $\mathrm{X}$-ray silicon detectors intended to be used for controlling low radiation doses specific to medical and synchrotron radiation applications. The critical steps of the planar process are the thermal oxidation of the silicon surface, the annealing of the implanted impurities and the gettering of contaminants. All these factors contribute to the magnitude of the reverse leakage current and consequently affect the stability of the detector after exposure to radiation and the sensitivity of its response. The fabricated test structures proved to be stable in X-ray radiation environments in the dose range 10-10 $\mathrm{Gy}$. The spectroscopic measurements demonstrated the possibility of use of these detectors for analyzing the X-ray spectrum of specific sources and for detecting low radiation levels.

\section{References}

[1] H. Struder, H. Soltau, Radiat. Protect. Dosimetry 61, 39 (1995).

[2] A. Thompson, in: Handbook for Synchrotron Radiation, Vol. 4, Eds. S. Ebashi, M. Koch, E. Rubenstein, Plenum Press, New York 1994, p. 583.

[3] D. Lazarovici, Proc. SPIE 2009, 56 (1993).

[4] E. Beuville, Nucl. Instrum. Methods Phys. Res. A 288, 68 (1990). 